\title{
Dinâmica do Banco de Sementes em Áreas com Aplicação FregÜENTE do HeRBICIDA GlyPHOSATE ${ }^{1}$
}

\author{
Seed Bank Dynamics in Areas with Frequent Glyphosate Application \\ MONQUERO, P.A. ${ }^{2}$ e CHRISTOFFOLETI, P.J. ${ }^{3}$
}

\begin{abstract}
RESUMO - Embora o herbicida glyphosate seja considerado não-seletivo, várias espécies de plantas daninhas apresentam certo grau de tolerância às doses recomendadas. No Brasil, já existem relatos de seleção de espécies de plantas daninhas tolerantes ao glyphosate em áreas com o uso intensivo deste herbicida. Assim, o objetivo desta pesquisa foi avaliar a influência da aplicação repetitiva do herbicida glyphosate sobre a dinâmica do banco de sementes das plantas daninhas tolerantes: Commelina benghalensis, Ipomoea grandifolia e Richardia brasiliensis; e das plantas daninhas suscetíveis: Amaranthus hybridus e Galinsoga parviflora. O experimento foi monitorado durante dois anos em áreas experimentais do Departamento de Produção Vegetal da Escola Superior de Agricultura "Luiz de Queiroz" Universidade de São Paulo, Piracicaba, São Paulo. Partindo de uma densidade de 100 plantas $\mathrm{m}^{-2}$ de cada espécie, o glyphosate foi aplicado periodicamente em pós-emergência sobre as plantas daninhas adultas nas doses de 0, 0,36, 1,44 e 2,88 kg e.a. ha ${ }^{-1}$. O banco de sementes do solo foi estimado através da avaliação de emergência direta das plântulas e extração física das sementes. O banco de sementes das plantas daninhas tolerantes ao glyphosate teve aumento significativo, principalmente nas menores doses. Por outro lado, o banco de sementes das espécies suscetíveis diminuiu ao longo do tempo mesmo nas menores doses do glyphosate. Na última análise do banco de sementes, as plantas daninhas tolerantes apresentaram, na maior dose de glyphosate aplicada, cerca de 12 milhões de sementes não-dormentes $\mathrm{ha}^{1}$, ao passo que as suscetíveis apresentaram cerca de 4 milhões de sementes não-dormentes hal. Concluiu-se que o uso intensivo do herbicida glyphosate pode ocasionar uma mudança na área, aumentando a freqüência das plantas tolerantes ao longo dos anos.
\end{abstract}

Palavras-chave: tolerância, Richardia brasiliensis, Commelina benghalensis, Ipomoea grandifolia, Galinsoga parviflora, Amaranthus hybridus.

\begin{abstract}
Although glyphosate is considered a non- selective herbicide, several weed species show a certain degree of tolerance to the recommended rates. Weed species tolerance to glyphosate in areas with intensive application of this herbicide has been reported in Brazil. Thus, the objective of this research was to evaluate the influence of the herbicide glyphosate on the seedbank dynamics of the tolerant weeds Commelina benghalensis, Ipomoea grandifolia and Richardia brasiliensis; and the susceptible weeds Amaranthus hybridus and Galinsoga parviflora. The experiment was monitored during two years in the experimental area of Department of Plant Production of ESALQ/University of São Paulo, Brazil. Beginning with an initial density of 100 plants $\mathrm{m}^{-2}$ of each species, glyphosate was sprayed periodically under post-emergence conditions at the rates of $0,0.36,1.44$ and $2.88 \mathrm{~kg}$ a.e/ ha. The seedbank in the soil was estimated through seedling emergence evaluation and physical extraction from the soil. Seedbank of the tolerant species increased specially at lower rates of glyphosate. On the other hand, the seedbank of susceptible species decreased during the experiment, even at lower rates. In the last seedbank evaluation, the tolerant weeds presented almost 12 million of seeds/ha, while the susceptible weeds showed about four million seeds/ha. It was concluded that the intensive use of glyphosate can cause a weed shift in the area, increasing the frequency of tolerant weeds along the years.
\end{abstract}

Key words: tolerance, Richardia brasiliensis, Commelina benghalensis, Ipomoea grandifolia, Galinsoga parviflora, Amaranthus hybridus.

Recebido para publicação em 23.8.2002 e na forma revisada em 2.4.2003.

Doutoranda em Agronomia, Dep. de Produção Vegetal, Universidade de São Paulo, Escola Superior de Agricultura "Luiz de Queiroz" - ESALQ-USP, Caixa Postal 09, 13418-900 Piracicaba-SP, Brasil, Bolsista da FAPESP, <pamonque@hotmail.com>;

${ }^{3}$ Professor Associado, Dep. de Produção Vegetal da ESALQ-USP.

Planta Daninha, Viçosa-MG, v.21, n.1, p.63-69, 2003 


\section{INTRODUÇÃO}

A denominação "Banco de Sementes" ou "Reservatório de Sementes" no solo tem sido usada na literatura internacional, segundo Carmona (1992), para descrever o montante de sementes não-dormentes e outras estruturas de propagação presentes no solo ou nos restos vegetais. Alguns pesquisadores estimaram que a quantidade de sementes presentes na camada arável do solo pode variar de 2.000 até 70.000 sementes $\mathrm{m}^{2}$ (Johnson \& Anderson, 1986) em diferentes agroecossistemas e localidades.

A utilização repetitiva de um mesmo herbicida ou de herbicidas com o mesmo mecanismo de ação e espectro de controle de plantas daninhas por diversos anos pode selecionar espécies tolerantes. Da mesma forma, herbicidas com efeito residual curto podem selecionar espécies com germinação tardia. Segundo Wilson (1998), o uso repetitivo de herbicidas com o mesmo espectro de ação na cultura do arroz por quatro anos tem conduzido à predominância da infestação de Eleocharis kuroguwae, Cyperus serotinus e Scirpus juncoides em supressão às plantas daninhas dicotiledôneas.

O glyphosate é um herbicida não-seletivo, de ação sistêmica, usado no controle de plantas daninhas anuais e perenes e, aparentemente, não tem atividade residual no solo (Rodrigues \& Almeida, 1998). Embora ainda não registrado no Brasil, o uso de culturas transgênicas resistentes ao glyphosate tem se expandido grandemente em alguns países, como Argentina e Estados Unidos.

Embora o glyphosate seja não-seletivo, espécies de plantas daninhas têm sido relatadas como tolerantes a este herbicida, como, por exemplo, Ambrosia artemisiifolia (Kapusta et al., 1994), Sesbania exaltatta, Ipomoea spp. (Jordan et al., 1997; Lich et al., 1997), Apocynum cannabium e Asclepsias syriaca (Wyrill \& Burnside, 1976). No Brasil, algumas espécies de plantas daninhas têm mostrado tolerância ao glyphosate (Durigan et al., 1988; Santos et al., 2001). Entretanto, a razão para a tolerância diferencial dessas espécies não é ainda compreendida.

Uma predição acurada da emergência de plantas daninhas do banco de sementes poderia permitir aos agricultores o controle mais eficiente, impedindo o uso inapropriado de herbicidas em pós-emergência (Cardina \& Sparrow, 1996). A estimativa qualitativa e quantitativa das sementes no banco de sementes é invariavelmente acompanhada pela germinação direta das amostras do solo e pela extração física das sementes seguida de ensaios de viabilidade (Luschei et al., 1998). Assim, foi conduzido este experimento com o objetivo de avaliar a influência da aplicação repetitiva do glyphosate em uma área sobre a dinâmica do banco de sementes das plantas daninhas Commelina benghalensis, Ipomoea grandifolia, Richardia brasiliensis, Amaranthus hybridus e Galinsoga parviflora.

\section{MATERIAL E MÉTODOS}

O experimento foi monitorado por dois anos em área experimental do Departamento de Produção Vegetal da Escola Superior de Agricultura "Luiz de Queiroz" - Universidade de São Paulo, Piracicaba, São Paulo. O delineamento experimental adotado foi o de blocos ao acaso com quatro tratamentos e oito repetições, com parcelas apresentando área útil de $2 \mathrm{~m}^{2}$. As parcelas foram tratadas com brometo de metila, a fim de se esterilizar o solo, eliminando assim o banco de semente preexistente.

As sementes das espécies daninhas Richardia brasiliensis, Commelina benghalensis e Ipomoea grandifolia, que são naturalmente tolerantes ao herbicida glyphosate nas doses recomendadas quando adultas, e sementes das espécies daninhas Galinsoga parviflora e Amaranthus hybridus, sensíveis à ação deste herbicida, foram semeadas no dia 16/9/2000. Após a germinação, as plântulas foram desbastadas, para obtenção da densidade aproximada de 100 plantas por parcela de cada espécie.

O herbicida glyphosate foi aplicado em pósemergência das plantas daninhas, antes da produção de sementes, com pulverizador costal pressurizado com $\mathrm{CO}_{2}$, com ponta de pulverização tipo leque de distribuição de jato elíptico, de ângulo de 80 e volume de calda de $300 \mathrm{~L}$ hal . As doses utilizadas do herbicida glyphosate foram de 0, 0,36, 1,44 e 2,88 kg e.a. ha ${ }^{1}$. As plantas daninhas que não foram controladas na primeira aplicação foram deixadas até a produção de sementes, sendo então 
incorporada toda a massa vegetal no solo. Em seguida, houve novo fluxo de emergência das plantas daninhas e nova aplicação de glyphosate em pós-emergência, antes da produção de novas sementes. Esses processos foram repetidos quatro vezes nas plantas A. hybridus G. parviflora e $R$. brasiliensis e três em $C$. benghalensise I. grandifolia. A época de aplicação do herbicida variou com o desenvolvimento de cada espécie de planta daninha.

O banco de sementes existente na área experimental foi monitorado durante dois anos de utilização de glyphosate a cada fluxo de emergência das plantas daninhas. As parcelas de cada tratamento foram avaliadas separadamente, determinando-se a emergência de plântulas em casa de vegetação e a separação física das sementes, métodos estes que serão descritos a seguir.

Inicialmente, foi avaliado o controle aos 14 dias após cada aplicação dos tratamentos com glyphosate em cada parcela de todas as espécies de plantas daninhas estudadas, usando escala de controle de 0 a 100 (controle nulo a total). Após a maturação das sementes das plantas escapes, eram realizadas amostragens de solo com um trado (cano de PVC adaptado) com $10 \mathrm{~cm}$ de diâmetro, sendo feitas coletas de $0-10 \mathrm{~cm}$ de profundidade do solo. Cada amostra foi composta por quatro subamostras, para se determinar a germinação direta de sementes em casa de vegetação, e quatro subamostras, para separação das sementes em laboratório. Foram, portanto, coletadas oito subamostras por parcela de campo a cada avaliação realizada. Depois de feitas as amostragens, houve a incorporação das sementes no solo em cada parcela.

O procedimento para quantificação de sementes em laboratório descrito a seguir foi baseado nos trabalhos de Buhler \& Maxwell (1993), com algumas modificações. Amostras provenientes do campo foram homogeneizadas e secas ao ar. Depois de seca, cada amostra foi peneirada em peneira de 10 mesh, retirando agregados e materiais inertes. A seguir, $150 \mathrm{~g}$ de amostra foram adicionados a uma solução de $50 \mathrm{~g}$ de $\mathrm{K}_{2} \mathrm{CO}_{3}$, dissolvidos em $200 \mathrm{~mL}$ de água. O carbonato de potássio foi utilizado, pois aumenta a densidade da solução e separa o material orgânico da parte mineral do solo. Uma vez que as amostras foram colocadas nesta solução em tubos de centrífuga com capacidade de $250 \mathrm{~mL}$, elas foram homogeneizadas por agitação manual por um minuto e posteriormente centrifugadas por 15 minutos a $10.000 \mathrm{~g}$. Os sobrenadantes foram retirados, passados por uma peneira de 50 mesh e colocados em um papel-filtro, para secagem preliminar. Depois, as sementes, juntamente com o material orgânico, foram colocadas em placas de Petri e secas em estufa a $35{ }^{\circ} \mathrm{C}$. Foi utilizado um soprador para retirar as partículas menos densas que as sementes. Posteriormente, as sementes de cada espécie estudada foram quantificadas, com a ajuda de um estereoscópio. Considerando-se que uma fatia de solo de um hectare igual a $10.000 \mathrm{~m}^{2}$, com $15 \mathrm{~cm}$ de profundidade, pesa em média $2.100 .000 \mathrm{~kg}$ (densidade do solo de $1,3 \mathrm{~g} \mathrm{~cm} \mathrm{~cm}^{3}$ ), portanto com $10 \mathrm{~cm}$ de profundidade, o peso é de $1.400 .000 \mathrm{~kg}$. O número provável de sementes $\mathrm{ha}^{1}$, a $10 \mathrm{~cm}$ de profundidade, foi calculado da seguinte forma:

$\frac{\text { Sementes extraídas }}{\text { Peso da amostra }(\mathrm{kg})}=\frac{\text { Número provável de sementes } / \mathrm{ha}}{1.400 .000 \mathrm{~kg}}$

Os resultados referentes às médias do número de sementes de cada espécie de planta daninha para cada tratamento foram submetidos à análise de variância, e as interações significativas tiveram suas médias comparadas pelo teste de Tukey $(\mathrm{P}<0,05)$.

Para verificar a porcentagem de sementes que germinam prontamente no banco de sementes, as amostras de $500 \mathrm{~g}$ de terra de solo coletadas na área experimental foram colocadas separadamente em bandejas com 8 $\mathrm{cm}$ de profundidade, em casa de vegetação com irrigação automática. Após cada fluxo de emergência, as plantas foram contadas e arrancadas, sendo a seguir feito um revolvimento do solo para estimular novos fluxos de emergência. A quantificação das sementes foi realizada até 21 dias após a instalação. O cálculo do número de sementes não-dormentes ha ${ }^{1}$ foi feito da seguinte maneira:

$\frac{\text { Número de plântulas }}{\text { Peso da amostra }(\mathrm{kg})}=\frac{\text { Número de sementes não dormentes } / \mathrm{ha}}{1.400 .000 \mathrm{~kg}}$

Os resultados referentes às médias de emergência em cada tratamento foram submetidos à análise de variância, e as interações significativas tiveram suas médias comparadas entre si pelo teste de Tukey $(\mathrm{P}<0,05)$.

Planta Daninha, Viçosa-MG, v.21, n.1, p.63-69, 2003 


\section{RESULTADOS E DISCUSSÃO}

A planta daninha A. hybridus é uma espécie daninha sensível à ação do glyphosate. Na Tabela 1 pode-se observar o controle eficaz deste herbicida sobre as plantas adultas, principalmente nas doses de $1,44 \mathrm{~kg}$ e.a. $\mathrm{ha}^{1}$ e 2,88 kg e.a. ha ${ }^{1}$. Observa-se na Figura $1 \mathrm{~A}$ a alta produção de sementes de A. hybridus - na última avaliação a testemunha apresentou cerca de 6,8 bilhões de sementes ha ${ }^{1}$. De acordo com Deuber (1992), Amaranthus spp, tem a capacidade de produzir até 120.000 sementes/ planta. À medida que a dose de glyphosate foi aumentada, o número de sementes foi decrescendo; na maior dose, o número de sementes nas avaliações variou de 431 milhões a 140 milhões de sementes hal ${ }^{1}$ Não foram realizados testes de viabilidades com as sementes extraídas, não podendo, portanto, ser consideradas como total de sementes viáveis. A Tabela 2 refere-se ao número de sementes não-dormentes de $A$. hybridus que germinam prontamente em casa de vegetação; os dados mostrados foram obtidos durante a última avaliação, realizada até 21 dias após a colocação das bandejas em casa de vegetação, e indicam que a testemunha apresentou 84 milhões de sementes não-dormentes $\mathrm{ha}^{1}$, diminuindo gradativamente com as doses aplicadas. $\mathrm{Na}$ maior dose do glyphosate foram encontradas 4,4 milhões de sementes não-dormentes hal. É importante lembrar que as sementes podem permanecer viáveis no solo por longo período sem germinar e que algumas sementes germinadas não conseguem emergir devido a condições ambientais desfavoráveis.
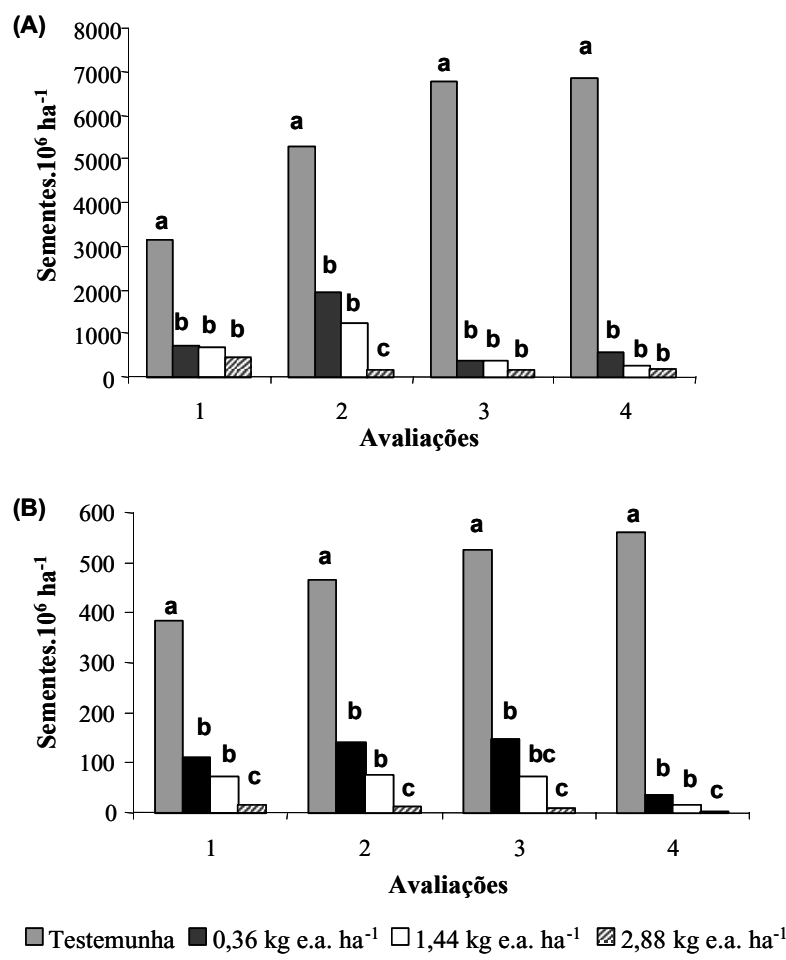

Figura 1 - Número de sementes $\left(10^{6} \mathrm{ha}^{-1}\right)$ de $A$. hybridus (A) e G. parviflora (B) em diferentes avaliações. Colunas com letras diferentes dentro de cada avaliação são estatisticamente diferentes entre si pelo teste de Tukey $(\mathrm{P}<0,05)$.

A planta daninha G. parviflora não é grande competidora em culturas que tendem ao fechamento, sendo altamente suscetível à ação do glyphosate (Kissmann \& Groth, 1992). O controle no campo desta planta foi satisfatório, oscilando de 70 a $90 \%$ (Tabela 1). O controle satisfatório desta planta é importante, pois é

Tabela 1 - Porcentagem de controle aos 14 dias após a aplicação do glyphosate, realizada antes da última avaliação de banco de sementes. Piracicaba-2002

\begin{tabular}{|l|c|c|c|c|c|}
\hline \multicolumn{1}{|c|}{ Tratamento } & AMACH $^{1 /}$ & IOGR $^{2 /}$ & GASPA $^{3 /}$ & RCHBR $^{4 /}$ & COMBE $^{5 /}$ \\
\hline Testemunha & $0,0 \mathrm{~d}$ & $0,0 \mathrm{~d}$ & $0,0 \mathrm{c}$ & $0,0 \mathrm{~d}$ & $0,0 \mathrm{c}$ \\
$0,36 \mathrm{~kg}$ e.a. $\mathrm{ha}^{-1}$ & $66,2 \mathrm{~b}$ & $26,2 \mathrm{c}$ & $76,2 \mathrm{~b}$ & $30 \mathrm{c}$ & $30,0 \mathrm{~b}$ \\
$1,44 \mathrm{~kg}$ e.a. $\mathrm{ha}^{-1}$ & $82,5 \mathrm{~b}$ & $35,0 \mathrm{~b}$ & $82,5 \mathrm{ab}$ & $42,5 \mathrm{~b}$ & $44,2 \mathrm{a}$ \\
$2,88 \mathrm{~kg}$ e.a. $\mathrm{ha}^{-1}$ & $95,0 \mathrm{a}$ & $52,50 \mathrm{a}$ & $92,5 \mathrm{a}$ & $81,2 \mathrm{a}$ & $50,5 \mathrm{a}$ \\
\hline CV\% & 7,3 & 12,1 & 8,2 & 13,9 & 11,6 \\
DMS (5\%) & 9,4 & 7,2 & 10,8 & 11,2 & 7,2 \\
\hline
\end{tabular}

${ }^{1 /} \mathrm{AMACH}=$ A. hybridus $;{ }^{2 /} \mathrm{IOGR}=$ I. grandifolia $;{ }^{3 /} \mathrm{GASPA}=$. parviflora $;{ }^{4 /} \mathrm{RCHBR}=R$. brasiliensis; $\mathrm{e}^{5 /} \mathrm{COMBE}=C$. benghalensis. Colunas com letras diferentes são estatisticamente diferentes entre si pelo teste de Tukey $(\mathrm{P}<0,05)$. 
uma espécie que pode desenvolver várias gerações por ano (Kissmann \& Groth, 1992). Em G. parviflora, o número de sementes encontradas nas amostragens foi relativamente menor (Figura 1B), pois sua capacidade de produção de sementes é menor que a de A. hybridus ela apenas apresentou alta produção de sementes na testemunha, com cerca de 560 milhões sementes ha ${ }^{1}$. Houve diferença estatística entre os tratamentos, e na maior dose de glyphosate a produção diminuiu para 2,3 milhões de sementes ha1.

De acordo com a Tabela 2, G. parviflora apresentou, na última avaliação, valores que variaram de 65 milhões de sementes nãodormentes $\mathrm{ha}^{1}$ na testemunha até 720.000 sementes não-dormentes $\mathrm{ha}^{1}$ na maior dose do herbicida, indicando que mesmo nas maiores doses as plantas remanescentes conseguiram produzir sementes suficientes para uma possível infestação da área.

A planta daninha I. grandifolia é uma planta infestante em lavouras, especialmente em culturas anuais de verão, nas regiões Sul, Sudeste e Centro-Oeste. Nos ensaios de campo o controle por glyphosate foi insatisfatório, ocorrendo apenas $52 \%$ de controle na maior dose (Tabela 1). Este resultado pode gerar alto acréscimo de sementes no banco de sementes do solo. Segundo Lorenzi (1994), esta é uma planta daninha capaz de produzir entre 5.000 e 6.000 sementes por planta, ocorrendo germinação até a profundidade de $10 \mathrm{~cm}$ no solo. $\mathrm{Na}$ Figura 2A pode-se observar a produção de sementes desta planta daninha, em que a testemunha alcançou os valores de 252 a
469 milhões de sementes hả. Na maior dose do glyphosate, essa produção, na última avaliação, diminuiu para 28 milhões sementes hal . Na Tabela 2, referente ao número de sementes não-dormentes, os dados obtidos durante 21 dias mostram que a testemunha apresentou 42 milhões de sementes não-dormentes $\mathrm{ha}^{1} \mathrm{e}$ a maior dose 12 milhões de sementes ha ${ }^{1}$, valor bem maior do que os produzidos pelas plantas suscetíveis ao glyphosate.
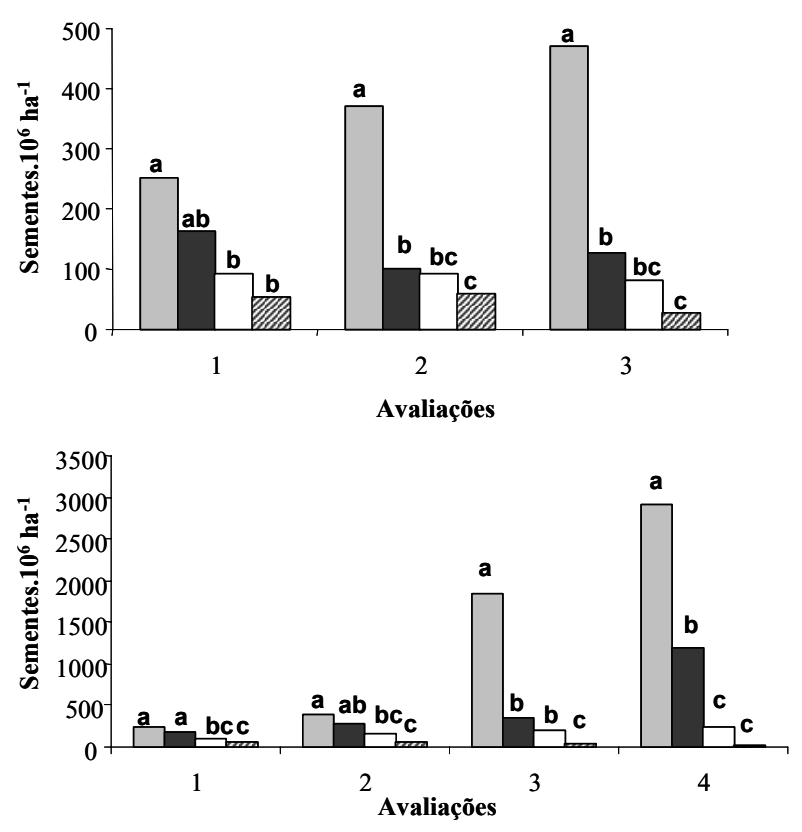

$\square$ Testemunha $\square 0,36 \mathrm{~kg}$ e.a. ha ${ }^{-1} \square 1,44 \mathrm{~kg}$ e.a. ha ${ }^{-1}$ 行,88 kg e.a. ha ${ }^{-1}$

Figura 2 - Número de sementes $\left(10^{6} \mathrm{ha}^{-1}\right)$ de I. grandifolia (A) e $R$. brasiliensis (B) em diferentes avaliações. Colunas com letras diferentes são estatisticamente diferentes dentro de cada avaliação entre si pelo teste de Tukey $(\mathrm{P}<0,05)$.

Tabela 2 - Número de sementes não-dormentes (milhões ha ${ }^{-1}$ ), ou seja, que emergiram das amostras coletadas 20 meses após a instalação do ensaio, nos diferentes tratamentos com glyphosate, 21 dias após a colocação em casa de vegetação

\begin{tabular}{|l|c|c|c|c|c|}
\hline \multicolumn{1}{|c|}{ Tratamento } & AMACH $^{1 /}$ & IOGR $^{2 /}$ & GASPA $^{3 /}$ & RCHBR $^{4 /}$ & COMBE $^{5 /}$ \\
\hline Testemunha & $84,0 \mathrm{a}$ & $42,0 \mathrm{a}$ & $65,2 \mathrm{a}$ & $81,2 \mathrm{a}$ & $30,8 \mathrm{a}$ \\
$0,36 \mathrm{~kg}$ e.a. $\mathrm{ha}^{-1}$ & $25,2 \mathrm{ab}$ & $22,4 \mathrm{a}$ & $28,0 \mathrm{ab}$ & $53,2 \mathrm{ab}$ & $28,1 \mathrm{a}$ \\
$1,44 \mathrm{~kg}$ e.a. $\mathrm{ha}^{-1}$ & $12,0 \mathrm{~b}$ & $19,6 \mathrm{a}$ & $16,8 \mathrm{~b}$ & $28,0 \mathrm{ab}$ & $18,4 \mathrm{ab}$ \\
$2,88 \mathrm{~kg} \mathrm{e.a.} \mathrm{ha}^{-1}$ & $4,4 \mathrm{~b}$ & $12,0 \mathrm{a}$ & $0,72 \mathrm{~b}$ & $8,4 \mathrm{~b}$ & $12,8 \mathrm{~b}$ \\
\hline CV\% & 85,5 & 49,1 & 78,1 & 61,0 & 29,2 \\
DMS (5\%) & 25,5 & 11,1 & 20,3 & 24,5 & 5,5 \\
\hline
\end{tabular}

${ }^{1 /} \mathrm{AMACH}=$ A. hybridus $;{ }^{2 /} \mathrm{IOGR}=$ I. grandifolia $;{ }^{\frac{3}{6}} \mathrm{GASPA}=$ G. parviflora $;{ }^{4 /} \mathrm{RCHBR}=R$. brasiliensis; $\mathrm{e}^{\frac{5}{2}} \mathrm{COMBE}=$ C. benghalensis. Colunas com letras diferentes são estatisticamente diferentes entre si pelo teste de Tukey $(\mathrm{P}<0,05)$. 
R. brasiliensis é uma planta daninha que, quando adulta, apresenta tolerância ao herbicida glyphosate (Lorenzi, 1994), o que pode ser observado na Tabela 1 . Na testemunha, a produção de sementes variou de 228 milhões a 2,9 bilhões (última avaliação) de sementes ha ${ }^{1}$; entretanto, na última avaliação feita na maior dose do herbicida foram constatados 28 milhões de sementes ha ${ }^{1}$ (Figura 2B). Com relação a sementes não-dormentes (Tabela 2 ), a testemunha obteve produção de 81 milhões de sementes hal no solo. Esta produção diminuiu com o aumento das doses; $\operatorname{com} 2,88 \mathrm{~kg}$ e.a. hà de glyphosate, houve a germinação de três plântulas, que representa 8,4 milhões de sementes não-dormentes $\mathrm{ha}^{1}$ - este valor, apesar de ser 10 vezes menor do que o obtido na testemunha, garante futuras infestações.

A planta daninha $C$. benghalensis é de difícil controle, principalmente na operação de dessecação para a semeadura de soja. Quando o glyphosate é aplicado sobre a planta adulta o controle pode ser insatisfatório, uma vez que a planta é pouco suscetível (Lorenzi, 1994). Além disso, é uma planta que pode produzir dois tipos de sementes - aéreas e subterrâneas - com capacidade de germinar e emergir desde cerca de 12 centímetros de profundidade (Rodrigues, 1992). No campo, a aplicação de glyphosate na maior dose resultou em controle de apenas 50\% (Tabela 1). A quantidade de sementes por 150 g de solo variou de 45 a 65 , ou seja, houve aumento de sementes de um fluxo para outro - isso mostra que, na última avaliação, na testemunha, havia cerca de 606 milhões de sementes hả e, na maior dose, 210 milhões (Figura 3). Na Tabela 2 pode-se ver que, na testemunha, o número de sementes não-dormentes foi de 30 milhões de sementes $\mathrm{ha}^{1} \mathrm{e}$, na maior dose, de 12,8 milhões de sementes hal. As sementes desta espécie podem ser oriundas da parte aérea ou da subterrânea e variar em tamanho e características de germinação e emergência.

Embora as sementes produzidas pelas plantas daninhas estudadas possam ser viáveis, a germinação foi reduzida, pois a aplicação do glyphosate na fase reprodutiva das plantas pode ter afetado os níveis do ácido indolacético (AIA) nas sementes e inibido a germinação e emergência das plântulas (Issacs et al., 1989).

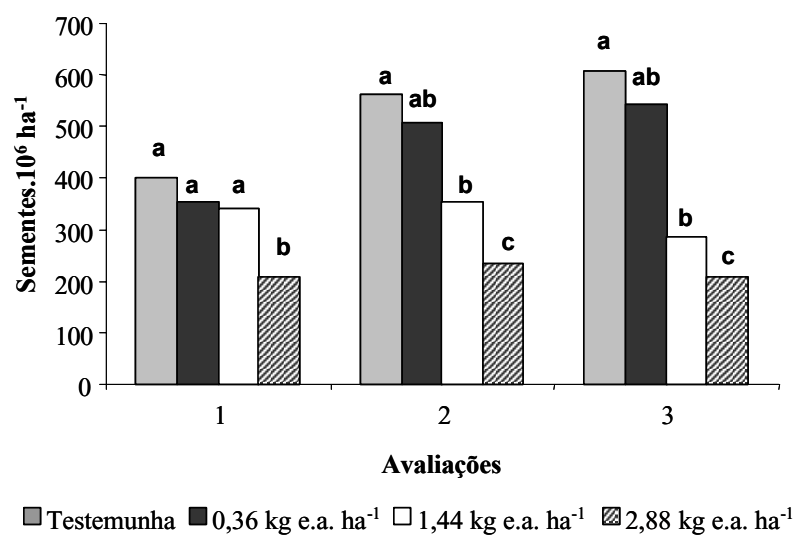

Figura 3 - Número de sementes $\left(10^{6} \mathrm{ha}^{-1}\right)$ de C. benghalensis em diferentes avaliações. Colunas com letras diferentes são estatisticamente diferentes dentro de cada avaliação entre si pelo teste de Tukey $(\mathrm{P}<0,05)$.

Os resultados indicam que o glyphosate aplicado repetidamente pode modificar a composição específica de plantas daninhas da área, levando à predominância de espécies tolerantes, como Commelina benghalensis, Ipomoea grandifolia e Richardia brasiliensis.

\section{AGRADECIMENTOS}

À FAPESP, pelo apoio financeiro na condução do trabalho, ao Departamento de Produção Vegetal da ESALQ/USP e aos funcionários Luiz Ferrari, Ony Rodrigues de Campos e, em especial, a Aparecido Mendes, pela grande ajuda na execução desta pesquisa.

\section{LITERATURA CITADA}

BUHLER, D. D.; MAXWELL, B. D. Seed separation and enumeration from soil using $\mathrm{K}_{2} \mathrm{CO}_{3}$ centrifugation and image analysis. Weed Sci., v. 41, n. 2, p. 298-302, 1993.

CARMONA, R. Problemática e manejo de banco de semente de invasoras em solos agrícolas. Planta Daninha, v. 10, n. 1, p. $5-13,1992$.

CARDINA, J.; SPARROW, D. H. A comparison of methods to predict weed seedling populations from the soil seedbank. Weed Sci., v. 44, n. 1, p. 46-51, 1996.

DEUBER, R. Botânica das plantas daninhas. In: DEUBER, R. Ciência das plantas daninhas. Jaboticabal: FUNEP, 1992. cap. 3, p. 31-73. 
DURIGAN, J. C; GALLI, A. J. B.; LEITE, G. J. Avaliação da eficiência da mistura de glyphosate e 2,4-D para o controle de plantas daninhas em citros. In: CONGRESSO BRASILEIRO DE HERBICIDAS E PLANTAS

DANINHAS, 17., 1988, Piracicaba. Resumos... Piracicaba: SBEHD, 1988. p. 303-304.

ISSACS, M. A. et al. Effects of late season herbicide application on Cassia obtusifolia seed production and viability. Weed Sci., v. 37, n. 6, p. 761-765.1989.

JOHNSON, R. G.; ANDERSON, R. C. The seed bank of tall grass prairie in Illinois. Am. Midland Natur., v. 115, p. $123-130,1986$.

JORDAN, D. L. et al. Influence of application variables on efficacy of glyphosate. Weed Tech., v. 11, n. 2, p. 354-362. 1997.

KAPUSTA, G.; KRAUZ, R. F.; MATTHEWS, J. L. Soybean tolerance and summer annual weed control with glufosinate and glyphosate in resistant soybean. Weed Sci., v. 49 , p. $120,1994$.

KISSMANN, K. G.; GROTH, D. Plantas infestantes e nocivas. São Paulo: BASF Brasileira, 1992. v. 2, 798 p.

LICH, J. M.; RENNER, K. A.; PENNER, D. Interaction of glyphosate with postemergence soybeans (Glycine max) herbicides. Weed Sci., v. 45, n. 1, p. 12-21, 1997.
LORENZI, H. Plantas daninhas do Brasil. 4.ed. Nova Odessa: Plantarum, 1994. 440 p.

LUSCHEI, E. C.; BUHLER, D. D.; DEKKER, J. H. Effect of separating giant foxtail (Setaria faberi) seeds from soil using potassium carbonate and centrifugation on viability and germination. Weed Sci., v. 46, n. 5, p. 545-548, 1998.

RODRIGUES, B. N. Estudos sobre a dormência, crescimento, absorção de macronutrientes e resposta à calagem por C. benghalensis L. 1992. 129 f. Tese (Doutorado em Produção Vegetal) - Universidade Estadual de São Paulo, Jaboticabal, 1992.

RODRIGUES, B. N.; ALMEIDA, F. S. Guia de herbicidas. Londrina: IAPAR, 1998. 648 p.

SANTOS, I. C. et al. Eficiência de glyphosate no controle de Commelina benghalensis e Commelina diffusa. Planta Daninha, v. 19, n. 1, p. 135-143, 2001.

WILSON, R. G. Biology of weed seed in the soil. In: ALTIERI, M. L.; LIEBMMAN, M. (Eds.). Weed Management in agroecosystem: ecological approaches. Boca Raton: CRS Press, 1998. p. 25-39.

WYRILL, J. B.; BURNSIDE, O. C. Absorption, translocation and metabolism of 2,4-D and glyphosate in common milkweed and hemp dogbane. Weed Sci., v. 24, n. 6 , p. 557-566, 1976 . 